Marcus Syring, Samuel Merk, Colin Cramer, Cansu Topalak \& Thorsten Bohl

\title{
Der Migrationshintergrund Lehramtsstudierender als Prädiktor ihrer Einstellungen zu heterogenen Lerngruppen
}

\section{Zusammenfassung}

Bildungspolitik und Bildungsadministration bemühen sich verstärkt um Lehrkräfte mit Migrationshintergrund. Es wird angenommen, dass diese Lehrkräfte einen positiven Effekt auf Schülerinnen und Schüler mit Migrationshintergrund haben oder zumindest günstigere Einstellungen gegenüber dem Umgang mit Heterogenität aufweisen. Diese Einstellungen sollen sich dann wiederum positiv auf die Unterrichtsgestaltung auswirken. Solche normativen Erwartungen spiegeln sich in unterschiedlichen nationalen wie internationalen Studien nur begrenzt wieder. Daher wird in diesem Beitrag der Frage nachgegangen, ob sich Lehramtsstudierende mit und ohne Migrationshintergrund hinsichtlich ihrer Einstellungen zum Umgang mit sozialer, ethnisch-kultureller sowie leistungsbezogener Heterogenität unterscheiden. Befragt wurden $N=877$ Studierende (169 mit Migrationshintergrund) an unterschiedlichen Hochschulen in Süddeutschland. Die Daten beider Substichproben (mit und ohne Migrationshintergrund) wurden gematcht und mit Random-Intercept-Modellen untersucht. Nach dem Matching zeigen sich unter den Studierenden mit Migrationshintergrund günstigere Einstellungen (geringere Kosten und negative Emotionen, höhere intrinsische Motivation und wahrgenommene Kompetenz) in Bezug auf alle drei Heterogenitätsformen. Die Ergebnisse werden mit Blick auf Implikationen für die Lehrerbildung diskutiert.

\section{Schlüsselwörter}

Migrationshintergrund, Lehrkräfte, Einstellungen, Heterogenität, Matching, Random-InterceptModelle

\section{Titel}

The migration background of teacher students as a predictor of their attitudes towards heterogeneous learning groups

\begin{abstract}
Education policy and administration are increasingly looking for teachers with migration background. They assume that these teachers have a positive effect on immigrant students or at least have more favorable attitudes toward dealing with heterogeneity. These attitudes should then have a positive effect on their teaching. Such normative expectations only could be partially confirmed in some national and international studies. Therefore, this paper examines the question of whether teacher students with or without a migration background differ in their attitudes towards dealing with social, ethnic-cultural and performance heterogeneity. $\mathrm{N}=877$ teacher students (169 with a migration background) have been interviewed at different universities in southern Germany. The data from both sub-samples (with and without migration background) were then matched and analyzed with Random Intercept Models. After matching, the results show more favorable attitudes (lower costs and negative emotions, higher intrinsic motivation and perceived competence) regarding all three forms of heterogeneity for students
\end{abstract}


with a migrant background. The results are finally discussed with regard to implications for teacher education.

\section{Keywords}

Migration background, teacher students, Attitudes, Heterogeneity, Matching, Random-Intercept models

\section{Einleitung}

Lehrpersonen sowie Lehramtsstudierende mit Migrationshintergrund werden von der Bildungspolitik stark umworben. Verantwortliche in der Bildungsadministration erhoffen sich so, Lehrpersonen zu gewinnen, die als Kulturvermittler und Rollenvorbilder für Schülerinnen und Schüler mit Migrationshintergrund fungieren (Rotter, 2012). Es wird auch angenommen, dass Lehrpersonen mit Migrationshintergrund aufgrund der eigenen Migrationserfahrung und der erfolgreichen eigenen Bildungsbiografie günstige Einstellungen mit Blick auf den Umgang mit Heterogenität in Unterricht und Schule haben (Magaldi, Conway \& Trub, 2018). Ob solche Annahmen berechtigt sind, die gegenwärtig bildungspolitische Entscheidungen mit steuern, und aus einer empirischen Sicht plausibel erscheinen, wird exemplarisch anhand der hier vorgestellten Studie diskutiert. Dazu wird geklärt, ob der Migrationshintergrund von Lehramtsstudierenden mit deren Einstellungen zu heterogenen Lerngruppen assoziiert ist. Zur Beantwortung dieser Forschungsfrage wird zunächst das methodische Vorgehen geschildert, anschließend werden die Ergebnisse dargestellt und mit Blick auf Implikationen für die Lehrerbildung diskutiert.

\section{Lehrpersonen mit Migrationshintergrund zwischen normativen Erwartungen und empirischen Realitäten}

Während an deutschen Schulen je nach Region ca. 30-40\% der Schülerinnen und Schüler einen Migrationshintergrund haben (Statistisches Bundesamt, 2017), stehen diesen verschiedenen Schätzungen zufolge auf Seiten der Lehrpersonen nur 6\% mit einem solchen gegenüber (Karakas, 2011; Georgi, 2011), nach Angaben des Statistischen Bundesamtes im Jahr 2008 sogar nur 4,7\% (Statistisches Bundesamt, 2008). Etwas ausgewogener zeigt sich das Bild mit Blick auf den Anteil von Lehramtsstudierenden mit Migrationshintergrund, der 12,5\% beträgt (Berthold \& Leichsenring, 2012). Grund genug, den Anteil von Lehrpersonen mit Migrationshintergrund zu steigern? Der Verband Bildung und Erziehung (2006) legte hierzu beispielsweise ein Positionspapier zur Notwendigkeit vor, mehr Lehrpersonen mit Migrationshintergrund einzustellen und auch der Nationale Integrationsplan (Presse- und 
Informationsamt der Bundesregierung, 2007) beinhaltet eine ähnliche Forderung. Zudem existieren besondere Förder- und Rekrutierungsprogramme für Lehramtsstudierende mit Migrationshintergrund. Auch international lassen sich solche besonderen Rekrutierungsbemühungen - bislang meist mit wenig Erfolg - beobachten (Kohli, 2018). Bislang verbinden sich mit dieser Forderung jedoch eher normative Erwartungen (Strasser \& Steber, 2009) als empirische Befunde.

\subsection{Normative Erwartungen}

An Lehrpersonen und pädagogisches Personal mit Migrationshintergrund werden besondere normative Erwartungen gestellt (Bräu et al., 2013), denn sie repräsentieren die Vielschichtigkeit der Gesellschaft und stellen auch Berührungs- und Bezugspunkte zu anderen Kulturen her. Sie fungieren somit als sogenannte Multiplikatoren bzw. Change Agents (Smith, 2015), die den Wandel innerhalb von Schule mit vorantreiben sollen. Gleichzeitig stellen sie ein identitätsstiftendes Vorbild (Smith, 2015) dar. Sie werden als Kulturbrückenbauer, Vermittler, Sprachübersetzer und Rollenvorbilder gesehen (Akbaba, Bräu \& Zimmer, 2013; Georgi, 2013; Georgi, Ackermann \& Karalas, 2011).

\subsection{Empirische Realitäten}

Wie bereits angedeutet, liegen bisher kaum empirische Befunde zur Frage der Bedeutsamkeit des Migrationshintergrundes von Lehramtsstudierenden vor. In Deutschland lassen sich nur einzelne und meist qualitative Studien zum Thema finden (Überblick bei Strasser \& Steber, 2009). Internationale Studien, vor allem seit den 1980er Jahren aus den USA und Großbritannien, lassen sich drei Forschungsschwerpunkten zuordnen (Karakasoglu, 2011): Es geht (1) um die Repräsentation und Rekrutierung von minority teachers, (2) um minority teachers als Mentorinnen und Mentoren, Rollenvorbilder und change agents oder (3) um Erfahrungen von Diskriminierung in der eigenen Ausbildung, in der Schule bzw. im Kollegium. Zentrale empirische Befunde dieser Forschung lassen sich thematisch gruppiert wie folgt zusammenfassen.

Bezüglich des wertschätzenden Klimas zeigen etwa Cunningham und Hargreaves (2007) in einer Studie für die USA, Großbritannien und die Schweiz, dass in Klassen mit Lehrpersonen aus Minderheiten dieses Klima der Wertschätzung gegenüber kultureller Vielfalt höher ausgeprägt ist. Für Deutschland kommt Edelmann (2008; 2013) in ihrer Interviewstudie zu dem Ergebnis, dass Lehrende mit Migrationshintergrund über eine stärker ausgeprägte interkulturelle Sensibilität verfügen. 
Georgi, Karakas und Ackermann (2011) haben in der Studie „Vielfalt im Lehrerzimmer“" Lehrpersonen mit Migrationshintergrund per Fragebogen und Interviews befragt (eine Vergleichsstichprobe fehlt hierzu jedoch). Sie konnten einen bewussten Umgang mit sprachlicher und kultureller Differenz aufgrund der eigenen biografischen Erfahrung nachweisen. Zudem konnten sie zeigen, dass Mehrsprachigkeit im Klassenzimmer angestrebt wird, auch wenn die eigene Herkunftssprache selten zum Einsatz kommt und ein besonderes Vertrauensverhältnis zwischen Lehrenden und Lernenden mit Migrationshintergrund vorherrscht. Lehrpersonen mit Migrationshintergrund zeigen ein besonderes Engagement für den Bildungserfolg von Kindern mit Migrationshintergrund, übernehmen häufig aber auch die Funktion eines Sozialarbeiters.

Von einem Teil der Lehrpersonen, so Georgi, Karakas und Ackermann (2011) weiter, wird die eigene Vorbildfunktion bejaht, von einem anderen Teil jedoch als Form von Zuschreibung abgelehnt. Zu diesem Ergebnis kommt auch Rotter (2012), der zufolge Lehrpersonen mit Migrationshintergrund mit ihrer Rolle als „Ausländerexperten“ oder „Migrantentanten“ eher hadern: „Gleichzeitig wollen jedoch Lehrkräfte mit Migrationshintergrund auch, das belegen einzelne empirische Untersuchungen, als selbstverständlicher Bestandteil des Lehrerkollegiums wahrgenommen und nicht auf eine Experten- und Botschafterrolle für Integration und Verständigung reduziert werden“ (Bandorski \& Karakasoglu, 2013, S. 134; siehe auch Karakasoglu, 2011; Otyakmaz, 2004). Auch international lässt sich dieser Befund beispielsweise in der qualitativen Studie von Henry (2018) mit zwölf US-amerikanischen Lehrkräften finden.

In der auf der Grounded Theory beruhenden Studie „I am Here for a Reason“ mit Lehramtsanfängern in den USA fanden Magaldi, Conwy und Trub (2018) heraus, dass minority teachers sich aufgrund der geteilten Erfahrungen eng mit Schülerinnen und Schülern mit Migrationshintergrund verbunden fühlen bzw. sich als Anwälte ihrer Interessen verstehen, jedoch dies nicht nur als Stärke sondern auch als besondere Herausforderung ansehen.

Ausgehend vom Modell der professionellen Kompetenz von Lehrkräften, welches den COACTIV-Studien zum Professionswissen von Lehrkräften, kognitiv aktivierendem Mathematikunterricht und der Entwicklung mathematischer Kompetenz zugrunde lag, untersuchten Hachfeld und Kollegen (2012) an 433 Mathematiklehramtsanwärterinnen und anwärtern Selbstwirksamkeitserwartungen, Enthusiasmus und Vorurteile für das Unterrichten von Schülerinnen und Schülern mit Migrationshintergrund in Abhängigkeit von ihrem eigenen Migrationshintergrund und ihren multikulturellen Überzeugungen. Die Ergebnisse ihrer Strukturgleichungsmodelle bestätigen dabei, dass Lehramtsanwärterinnen und -anwärter mit 
Migrationshintergrund über ausgeprägtere multikulturelle Überzeugungen verfügen, was wiederum ihre Selbstwirksamkeitserwartungen und ihren Enthusiasmus positiv sowie ihre Vorurteile negativ beeinflussten.

Neugebauer und Klein (2016) untersuchten anhand von Daten des Nationalen Bildungspanels (NEPS), inwieweit Kinder mit Migrationshintergrund von der Erziehung von Deutschen bzw. Erziehern mit Migrationshintergrund bezogen auf Vorläuferkompetenzen in Sprache, Mathe und Naturwissenschaften sowie die Sozialkompetenz profitieren. Die Ergebnisse zeigen dabei keine höheren Kompetenzen von Kindern mit Migrationshintergrund, die von Erzieherinnen und Erziehern mit Migrationshintergrund betreut wurden. Auch zeigt sich kein positiver Effekt einer solchen Betreuung auf das Kooperationsverhalten der Eltern dieser Kinder.

Edelmann (2008, 2013) interviewte Grundschullehrkräfte, darunter auch 15 mit Migrationshintergrund. Anschließend identifizierte sie Typen mit Blick auf deren Einstellungen zur migrationsbedingten Heterogenität in einem Spektrum vom abgrenzenddistanzierendem bis hin zum kooperativ-synergieorientiertem Typ. Sie fand dabei bis auf den distanzierenden Typ Lehrpersonen mit Migrationshintergrund in allen Typen wieder. Darin kann ein Anzeichen gesehen werden, dass der Migrationshintergrund tendenziell ein Hinweis auf eine (als normativ angemessen geltende) Einlassung auf entsprechende Schülerinnen und Schüler ist.

Zusammenfassend lässt sich festhalten, dass die empirische Befundlage knapp und inkonsistent ist. Lehrpersonen mit Migrationshintergrund scheinen zwar „ein Schlüssel zur interkulturellen Schulentwicklung in Deutschland [darzustellen], [taugen] aber nicht zum Allheilmittel gesellschaftlicher Integration“ (Georgi et al., 2011, S. 207). Der Migrationshintergrund einer Lehrkraft wird als Qualitätsmerkmal jedoch oftmals überbewertet und zeigt sich bisher nicht in den empirischen Daten oder verdeutlicht eine diffuse empirische Befundlage. „Der ,Migrationshintergrund“ für sich allein genommen bedeutet noch nicht eine automatische Ausprägung von ,interkultureller Kompetenz', auch wenn davon auszugehen ist, dass im Bereich der Empathie und Perspektivenübernahme gute Voraussetzungen durch eigene, biografische Voraussetzungen gegeben sein können“ (Karaksoglu, 2011, S. 131). Daher bleibt die Frage offen, ob der Migrationshintergrund sich beispielsweise vorteilhaft auf die Einstellungen zum Umgang mit Heterogenität auswirken kann und falls ja, einen positiven Effekt auf die Unterrichtsgestaltung hat.

\section{Einstellungen von Lehrpersonen zu heterogenen Lerngruppen}


Die Untersuchung von Einstellungen zu heterogenen Lerngruppen ist deshalb von Bedeutung, weil Einstellungen von Lehrerinnen und Lehrern als „wichtige handlungsregulierende Faktoren“ gelten, sie „beeinflussen die Wahrnehmung“ (Kunz, Luder \& Moretti, 2010, S. 84; vgl. auch Ajzen \& Fishbein, 2000; Fives \& Buehl, 2012). Bereits Pajares (1992) bezeichnet Einstellungen als Filter der Wahrnehmung. So können in Bezug auf (künftige) Lehrpersonen Einstellungen als „Vorstellungen und Annahmen [...] über schul- und unterrichtsbezogene Phänomene und Prozesse mit einer bewertenden Komponente“ (Kunter \& Pohlmann, 2009, S. 267) definiert werden. Bezüglich der Einstellungen werden eine kognitive, eine motivationalaffektive und eine behaviorale Komponente unterschieden (Eagly \& Chaiken, 1993; Rosenberg \& Hovland, 1960), die potenziell alle für das Lehrerhandeln von Relevanz sind. Viele Instrumente zur Erfassung von Einstellungen (nicht nur zur Heterogenität) bilden nur die kognitive Komponente ab (Syring, Tillmann, Weiß \& Kiel, 2018). Das in dieser Studie verwendete Instrument von Gebauer, McElvany und Klukas (2013) trägt der Idee von Eagly und Chaiken (1993) Rechnung, indem es die Einstellungen über mehrere Dimensionen erfasst (siehe auch Methode). Vermittelt über die Unterrichtsplanung und -gestaltung haben Einstellungen einen Effekt auf den Lernerfolg der Schülerinnen und Schüler (Avramidis \& Norwich, 2002), wie beispielsweise Untersuchungen von Voss, Kleickmann, Kunter und Hachfeld (2011) im Rahmen der COACTIV-Studie für das Fach Mathematik oder von Hartinger, Kleickmann und Hawelka (2006) für die Nutzung Offenen Unterrichts zeigen. Aus professionstheoretischer Sicht werden Einstellungen in einem kompetenzorientierten Verständnis neben Professionswissen, selbstregulativen Fähigkeiten und motivationalen Orientierungen als eine von vier zentralen Dimensionen professioneller Handlungskompetenz angenommen (siehe z.B. Bosse \& Spörer, 2014; Kunz et al., 2010).

Die Frage nach Einstellungen von Lehrerinnen und Lehrern zu heterogenen Lerngruppen (insbesondere auch zur Inklusion) stellt einen Spezialfall der Forschung zu Einstellungen dar. Mit der Konjunktur des Heterogenitäts-Diskurses im Allgemeinen (vgl. z.B. den zuletzt erschienenen Sammelband von Bohl, Budde \& Rieger-Ladich, 2017) zeigt sich auch ein verstärkter Fokus auf Einstellungen zu Heterogenität in der Professions- und Unterrichtsforschung (Hartwig, Schwabe, Gebauer \& McElvany, 2017; Heyl, Janz, Seifried \& Trumpa, 2014; Kopmann \& Heinz, 2016).

\subsection{Prädiktoren von Einstellungen zum Umgang mit Heterogenität}

Aufgrund der Bedeutung der Einstellungen von Lehrpersonen zu heterogenen Lerngruppen stellt sich die Frage, inwiefern diese von personalen Merkmalen Lehramtsstudierender 
abhängen: Haben die individuellen Eingangsvoraussetzungen ein erklärendes Potenzial für die handlungsrelevanten heterogenitätsbezogenen Einstellungen? In der Literatur gelten an die Person gebundene Merkmale als moderierende Variablen im gesamten Verlauf der Wirkungskette von Lehrerbildung. Sie haben Einfluss sowohl auf die Nutzung von Angeboten der Lehrerausbildung, auf den Aufbau professioneller Kompetenz sowie auf den Grad der Performanz, die Lehrpersonen beim Unterrichten zeigen (z.B. Kunter et al., 2011; Voss et al., 2015; Cramer, 2016). Es wird angenommen, dass Lehramtsstudierende auch das heterogenitätsbezogene Lernangebot, das ihnen im Studium unterbreitet wird, in Abhängigkeit von individuellen Eingangsvoraussetzungen nutzen können und dass sie in der Folge mehr oder weniger einschlägige Kompetenz erwerben, die von Relevanz für das Lehrerhandeln und somit das Schülerlernen sein können. Als personale Merkmale, die von Bedeutung für den Kompetenzerwerb und das Lehrerhandeln sind, werden u.a. Persönlichkeitsmerkmale und Interessen oder Leistungsmerkmale, aber auch die hier in Rede stehenden Herkunftsmerkmale Lehramtsstudierender angenommen (Cramer, 2016). Letztere dürften vor allem mit Blick auf die ethnisch-kulturelle und soziale Heterogenität im Zusammenhang mit eigener Migrationserfahrung stehen und von Relevanz für diesbezügliche Einstellungen sein. Dieser weitgehend ungeklärten Annahme geht dieser Beitrag nun empirisch nach.

\subsection{Einstellungen zu ethnisch-kultureller, sozialer und Leistungsheterogenität}

Die Offenheit des Begriffs der Heterogenität ist Stärke und Schwäche zugleich (Budde, 2012): Er bündelt verschiedene Differenzlinien bzw. Formen von Vielfältigkeit, vermengt sie dadurch aber auch stark. In den letzten Jahren stehen in der Schul- und Unterrichtsforschung v.a. drei Formen von Heterogenität im Fokus: die ethnisch-kulturelle, die soziale und die Leistungsheterogenität. Zum Bereich der Leistungsheterogenität lassen sich auch einige sonderpädagogische Förderbedarfe zählen. Aufgrund der Diskurse um diese drei Formen (siehe auch Budde, 2012) werden die Einstellungen in Bezug auf diese in der berichteten Studie untersucht. Dabei wird unter den drei Formen verstanden:

- Ethisch-kulturelle Heterogenität: Unterschiedlichkeit von Schülerinnen und Schülern mit Blick auf ihren kulturellen Hintergrund (Migrationsgeschichte, Erst-/Zweitsprache, kulturelle und religiöse Zugehörigkeit etc.)

- Soziale Heterogenität: Unterschiedliches Ausmaß an sozialem Kapital führt zu herkunftsbedingten Unterschieden, die wiederum von Relevanz für die Leistungen von Schülerinnen und Schülern sein können (Bourdieu, 2012) 
- Leistungsheterogenität: unterschiedliche Leistungsausprägung trotz gleichen Alters und gleicher Bildungsstufe (Blossfeld et al., 2007)

Ein weiterer Grund für den besonderen Fokus auf diese drei Formen liegt auch darin, dass diese oft auch als Prädiktoren, Kovariaten oder Korrelate in Verbindung mit schulischen Leistungen auftreten (z.B. Baumert \& Lehmann, 1997; Bos et al., 2007; Prenzel et al., 2006).

\section{Fragestellung}

Wie im theoretischen Teil aufgezeigt (Kap. 2.), besteht eine Diskrepanz zwischen den normativen Erwartungen an Lehrpersonen mit Migrationshintergrund einerseits und der empirischen Evidenz zu dessen Relevanz. Der Beitrag prüft daran anschließend folgende Forschungsfrage: Inwiefern geht das Herkunftsmerkmal Migrationshintergrund mit differentiellen Einstellungen zu heterogenen Lerngruppen (als ein Teilaspekt professioneller Kompetenz) einher? Als Hypothese wird angenommen, dass Lehramtsstudierende mit Migrationshintergrund günstigere Einstellungen aufweisen.

\section{Methode}

\subsection{Stichprobe und Anlage der Studie}

Zur Beantwortung der Forschungsfrage wurden $N=877$ Lehramtsstudierende aus sechs Hochschulstandorten in Baden-Württemberg (davon 622 weiblich; 432 an Universitäten und 445 an Pädagogischen Hochschulen eingeschrieben; 169 Studierende mit Migrationshintergrund) befragt. Die paper-pencil-Erhebung fand jeweils im Rahmen einer Pflichtveranstaltung für Lehramtsstudierende statt, in der TestleiterInnen präsent waren und die Befragung selbst durchführten. Die Teilnahme war freiwillig und wurde nicht belohnt. Da das Merkmal Migrationshintergrund in Deutschland mit differentiellen sozioökonomischen und demografischen Merkmalen assoziiert ist (Bildungsberichterstattung, 2016; Schäfer \& Brückner, 2008), müssen diese Merkmale bei der Betrachtung prädiktiver Effekte des Migrationshintergrundes kontrolliert werden. Dies geschieht anhand eines one-to-one Matching-Verfahrens (siehe 4.3 Statistische Verfahren und Analysen), das jedem Lehramtsstudierenden mit Migrationshintergrund einen in allen berücksichtigten Kovariaten ähnlichen Lehramtsstudierenden ohne Migrationshintergrund zuordnet. Die Modellierung des prädiktiven Effektes des Migrationshintergrundes auf die Überzeugungen zu heterogenen Lerngruppen erfolgt dann anhand des so generierten, balancierten Datensatzes. 


\subsection{Instrumente}

Die Einstellungen der Lehramtsstudierenden zu heterogenen Lerngruppen wurden mit einem Instrument von Gebauer, McElvany und Klukas (2013) erfasst, welches bereits auf seine faktorielle Validität hin überprüft wurde (Merk, Cramer, Dai, Bohl \& Syring, 2018). Dieses Instrument (siehe auch Tabelle 1) erfasst jeweils fünf Dimensionen von Einstellungen (Wert, Kosten, negative Emotionen, intrinsische Motivation und wahrgenommene Kompetenz mit Blick auf heterogene Lerngruppen) für jeweils drei Formen von Heterogenität (soziale, ethnisch-kulturelle und leistungsbezogene).

Tabelle 1. Struktur des Instrumentes von Gebauer, McElvany und Klukas (2013).

\begin{tabular}{|c|c|c|c|c|c|c|}
\hline & \multicolumn{5}{|c|}{ Einstellungen } \\
\hline & & \multicolumn{2}{|c|}{ kognitiv } & \multicolumn{2}{|c|}{ motivational-affektiv } & behavioural \\
\hline & & Wert & Kosten & $\begin{array}{c}\text { negative } \\
\text { Emotionen }\end{array}$ & $\begin{array}{l}\text { intrinsische } \\
\text { Motivation }\end{array}$ & $\begin{array}{c}\text { wahrgenommene } \\
\text { Kompetenz }\end{array}$ \\
\hline \multirow{3}{*}{ Ẽ } & sozial & 5 Items & 5 Items & 5 Items & 5 Items & 5 Items \\
\hline & ethnisch-kulturell & 5 Items & 5 Items & 5 Items & 5 Items & 5 Items \\
\hline & leistungsbezogen & 5 Items & 5 Items & 5 Items & 5 Items & 5 Items \\
\hline
\end{tabular}

Die Daten zu den Überzeugungen zu heterogenen Lerngruppen weisen also eine geschachtelte Struktur auf: Jedes Item wurde von jedem Lehramtsstudierenden dreifach beantwortet (für jede Heterogenitätsform einmal). Um die faktorielle Struktur des Instrumentes zu prüfen, wurden deshalb konfirmatorische Mehrebenenfaktorenanalysen (MCFA, Hox, 2010; Mehta \& Neale, 2005) herangezogen. Ein Modell mit $\tau$-kongenerischen Messmodellen und drei bzw. acht (anhand von Modifikationsindices ausgewählten) Residualkovarianzen auf beiden Ebenen resultierte in einer guten Modellanpassung $\left(\chi^{2}=1718.51, \mathrm{df}=519, \mathrm{CFI}=0.939\right.$, TLI $=0.929$, RMSEA $=0.03$, SRMRwithin $=0.051$, SRMRbetween $=0.087)$. Gemäß dieses Messmodells wurde anschließend die Reliabilität mit McDonalds $\omega$ geschätzt (siehe Tabelle 2).

Tabelle 2. Wertebereiche der Punktschätzungen sowie über die imputierten Datensätze hinweg vereinigte $95 \%$ Konfidenzintervalle (95\% KI) der Skalenreliabilität.

\begin{tabular}{|c|c|c|}
\hline Dimension & Beispielitem & $\begin{array}{r}\text { McDonalds } \omega \\
(95 \% \mathrm{KI})\end{array}$ \\
\hline Wert & $\begin{array}{l}\text { Schüler/innen profitieren durch Unterricht in kulturell/leistungs-/ sozial } \\
\text { heterogenen Lerngruppen hinsichtlich ihres schulischen Lernens. }\end{array}$ & $\begin{array}{r}.65<\omega<.75 \\
{[.60, .78]}\end{array}$ \\
\hline Kosten & $\begin{array}{l}\text { Die kulturelle/ leistungsbezogene/ soziale Heterogenität in unseren } \\
\text { Schulkassen erfordert von den Lehrkräften einen zusätzlichen Arbeitsaufwand. }\end{array}$ & $\begin{array}{r}.72<\omega<.79 \\
{[.69, .81]}\end{array}$ \\
\hline negative Emotionen & $\begin{array}{l}\text { Im Hinblick auf das Unterrichten von kulturell/leistungs-/sozial heterogenen } \\
\text { Klassen fühle ich mich angespannt bei dem Gedanken daran, die Kinder } \\
\text { entsprechend ihren kulturellen/ leistungsbezogenen/ sozialen Voraussetzungen } \\
\text { zu unterrichten }\end{array}$ & $\begin{array}{r}.81<\omega<.88 \\
\quad[.78, .90]\end{array}$ \\
\hline
\end{tabular}




\begin{tabular}{lll}
$\begin{array}{l}\text { intrinsische } \\
\text { Motivation }\end{array}$ & $\begin{array}{l}\text { Im Hinblick auf das Unterrichten von kulturell/sozial/ leistungsheterogenen } \\
\text { Klassen weiß ich, dass ich es schaffen werde, die erwarteten Lerninhalte zu } \\
\text { vermitteln, wenn ich mich darum bemühe. }\end{array}$ \\
$\begin{array}{l}\text { wahrgenommene } \\
\text { Kompetenz }\end{array}$ & $\left.\begin{array}{l}\text { Die kulturelle/leistungsbezogene/soziale Heterogenität in unseren } \\
\text { Schulklassen wird mir beim Unterrichten Spaß machen }\end{array}\right] .86<\omega<.87$ \\
\hline
\end{tabular}

Die personalen bzw. herkunftsbezogenen Merkmale wurden wie folgt operationalisiert:

Der Migrationshintergrund der Studierenden wurde mit Hilfe eines dichotomen Items erhoben. Dabei wurden diejenigen Befragten als angehende Lehrpersonen mit Migrationshintergrund definiert, die der Fragen nach der Herkunft der Eltern mit den Antwortoptionen „ein Elternteil ist in Deutschland geboren, der andere nicht“ bzw. „sind beide nicht in Deutschland geboren“ zustimmten. Wer hingegen die Option „sind beide in Deutschland geboren“wählte, wurde als Befragte/Befragter ohne Migrationshintergrund definiert. Damit folgt die Erfassung und Definition des Migrationshintergrundes dem Vorgehen des Statistischen Bundesamtes (2017).

Der sozioökonomische Status wurde durch offene Fragen erfasst: „Welchen Beruf übt ihre Mutter/ihr Vater gegenwärtig aus? oder In welchem Beruf hat ihre Mutter/ihr Vater zuletzt gearbeitet?". Die Antworten wurden nach der International Standard Classification of Occupations (ISCO) kodiert. Auf dieser Grundlage konnte der International Socio-Economic Index of Occuptional Status (HISEI, Ganzeboom, De Graaf, Treiman \& De Leeuw) gebildet werden.

Als Indikator für das soziokulturelle Milieu wurde die Anzahl der Bücher anhand eines Single-Items (,, Wie viele Bücher gibt es in Ihrer Familie ungefähr? (ohne Zeitschriften, Zeitungen oder Schulbücher)“; gruppiertes Antwortformat: 0-10, 11-25, 26-100, 101-200, mehr als 200) erfasst.

Sowohl der sozioökonomische Status als auch das soziokulturelle Milieu wurden aufgrund des vermuteten Zusammenhangs mit dem Migrationshintergrund erhoben (Sachverständigenrat deutscher Stiftungen für Integration und Migration, 2016), um eine Konfundierung des Effektes des Migrationshintergrundes zu vermeiden.

Als weitere Kovariate wurde mit zwei Single-Likert-Items (,Wie oft haben Professor/innen oder Dozent/innen in Veranstaltungen über Heterogenität gesprochen?"; „Wie oft hat Ihr Mentor/Ihre Mentorin in Schulpraktika über Heterogenität gesprochen?") erfasst, inwiefern die Befragten die Frage nach heterogene Lerngruppen bereits in der Lehrerbildung thematisiert hatten.

Schließlich wurde die Kontakthäufigkeit der Lehramtsstudierenden mit Menschen aus anderen sozialen Milieus, mit anderer ethnischer Herkunft oder mit Behinderung anhand von 
Likert-Items erhoben (Beispielitem: ,, Wie oft haben Sie momentan Kontakt mit Menschen aus anderen Kulturen?").

\subsection{Statistische Verfahren und Analysen}

\section{Matching}

Der Migrationshintergrund stellt eine soziodemographische Variable dar, die nicht experimentell variiert werden kann. Dieser Umstand führt zu einigen Herausforderungen bei der Untersuchung kausaler oder prädiktiver Effekte dieser Variable. Vorliegend etwa ist zu erwarten, dass neben dem Migrationshintergrund auch andere soziodemografische Variablen wie z.B. der sozioökonomische Status oder das Vorwissen zu Kompositionseffekten mit Einstellungen $\mathrm{zu}$ heterogenen Lerngruppen assoziiert sind. Eine effiziente Methode zur simultanen Kontrolle vieler Kovariaten stellen multivariate Matching-Verfahren dar (Adelson, 2013). Jeder Untersuchungseinheit der Treatmentgruppe (hier Studierende mit Migrationshintergrund) wird dabei eine Untersuchungseinheit der Kontrollgruppe (hier Studierende ohne Migrationshintergrund) zugeordnet, welche einander in allen Kovariaten möglichst ähnlich sind. So resultiert ein Datensatz reduzierter Größe, in welchem die Kovariaten in beiden Gruppen die gleiche Verteilung aufweisen.

Es existieren eine Reihe von semi- und nichtparametrischen Verfahren für diese Art vorbereitender Datenanalyse (Ho et al., 2011). Diese Verfahren können zunächst nach dem Distanzmaß klassifiziert werden, welches die Ähnlichkeit zweier Sets von Kovariaten (trotz unterschiedlicher Skalenniveaus und Metriken dieser) eindimensional ausdrückt. Hier werden in den Sozialwissenschaften insbesondere Propensity-Scores zunehmend eingesetzt (Thoemmes \& Kim, 2011).

Neben einer Klassifizierung nach dem verwendeten Distanzmaß können MatchingVerfahren auch nach dem eigentlichen Prozess des Zuordnens der Einheiten aus den beiden Gruppen zueinander systematisiert werden. Diese unterscheiden sich beispielsweise in der Anzahl einander zugeordneter Untersuchungseinheiten, deren einfachen oder mehrfachen Verwendung oder ihrer zugelassenen maximalen Distanz (für eine Übersicht siehe Ho et al., 2007).

Vorliegend wurde ein one-to-one Nearest-Neighbor-Matching anhand von PropensityScores durchgeführt. Dabei wurde jeder Untersuchungseinheit der Treatmentgruppe (beginnend mit dem höchsten Propensity-Score) genau eine Untersuchungseinheit der 
Kontrollgruppe zugeordnet. Die Untersuchungseinheiten der Kontrollgruppe wurden einmalig verwendet.

\section{Prädiktive Effekte}

Die hier untersuchten Einstellungen zu heterogenen Lerngruppen ergeben aufgrund der Anlage des Instrumentes (siehe Tabelle 1) eine genestete Datenstruktur: Die Items jeder Einstellungsdimension (Wert, Kosten, negative Emotionen, intrinsische Motivation, wahrgenommene Kompetenz) sind bei der Erfassung der Einstellungen für die drei Arten von Heterogenität dieselben; man könnte also auch von einem Within-person-Experiment mit dem Faktor „Art der Heterogenität“ sprechen. Die aus diesem Design resultierenden Abhängigkeiten der Daten müssen in der Modellierung prädiktiver Effekte des Migrationshintergrundes berücksichtigt werden, da eine Missachtung unter anderem zu verzerrten Standardfehlerschätzungen führen kann (Dorman, 2008).

Vorliegend wurden die prädiktiven Effekte des Migrationshintergrundes auf die Überzeugungen zu heterogenen Lerngruppen mit Random-Intercept Modellen untersucht. Zur Schätzung der Parameter wurde das Paket lme4 (Bates, Maechler, \& Bolker 2013) der statistikorientierten, freien und quelloffenen Programmierumgebung R (R Core Team, 2014) verwendet.

\section{Fehlende Werte}

Der vorliegende Datensatz enthält in den dafür typischen Variablen fehlende Werte (z.B. HISEI: $21.2 \%$ ). Um damit potentiell verbundene Probleme verzerrter Parameterschätzungen (Graham, Cumsille \& Elek-Fisk, 2003) zu vermeiden, wurde den fehlenden Werten mit einem auf Chained Equations basierenden Verfahren multipler Imputation begegnet. Bei diesen werden die fehlenden Werte durch mehrere (hier sieben) sogenannte plausible Werte ersetzt, die sich in der Regel auch voneinander unterscheiden. Die Schätzung der Propensity Scores und der prädiktiven Effekte erfolgten dann getrennt auf den vollständigen Datensätzen. Die Ergebnisse der einzelnen Modellparameterschätzungen wurden dann nach den Regeln von Rubin (1987) zu sogenannten gepoolten Parametern zusammengefasst.

\section{Ergebnisse}

Nachfolgend wird zunächst die Qualität des Propensity Score Matching berichtet (5.1.), im Anschluss werden die prädiktiven Effekte des Migrationshintergrundes dargestellt (5.2). 


\subsection{Qualität des Propensity Score Matching}

Für die Qualität der Analysen ist von zentraler Bedeutung, die Balance der Kovariaten vor und nach dem Propensity-Score Matching zu betrachten: Abbildung 1 stellt arithmetische Mittel und Standardabweichungen entsprechend differenziert dar.

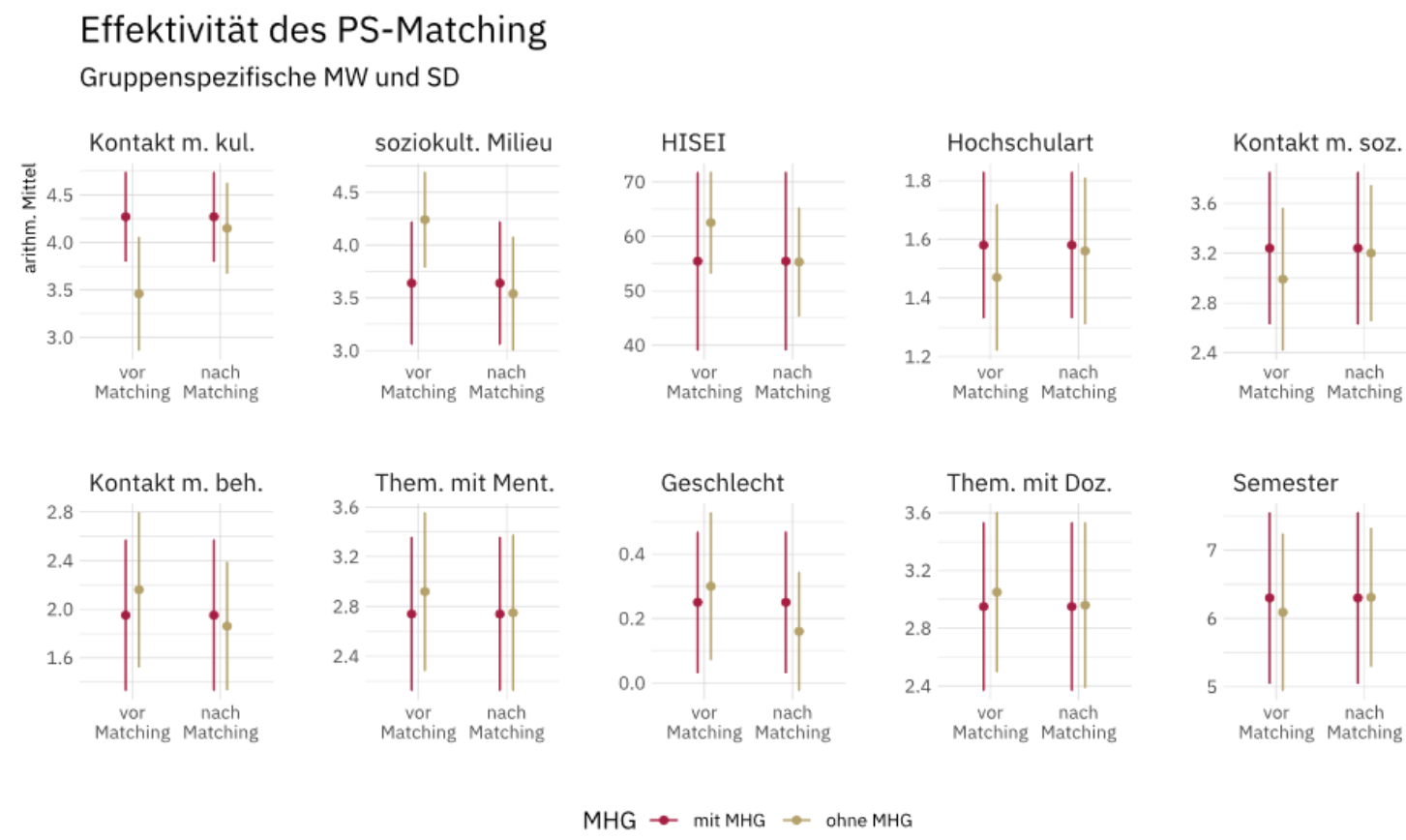

Abb. 1. Arithmetische Mittel und Standardabweichungen $( \pm 1)$ der in das Matching eingehenden Kovariaten. Subplots sortiert nach der Effektstärke der Mittelwertsunterschiede vor dem Matching (Cohen's d). MHG = Migrationshintergrund, Kontakt m. kult. = Kontakthäufigkeit mit Menschen anderer kultureller Herkunft, soziokult. Milieu = soziokulturelles Milieu; Kontakt m. soz. = Kontakthäufigkeit mit Menschen anderer sozialer Milieus, Them. mit Ment. = Häufigkeit der Thematisierung mit Mentor/Mentorin, Them. mit Doz. = Häufigkeit der Thematisierung mit Dozent/Dozentin.

Das Minium aller bootstrapbasierten $p$-Werte von $p=.071$ für die Kolmogorov-Statistik nach dem Matching (über alle Imputationen und Variablen hinweg; vor dem Matching vier hochsignifikante $p$-Werte) zeigt, liefert Evidenz dafür, dass die Kovariaten nach dem Matching nicht nur in den Mittelwerten, sondern bzgl. der gesamten Verteilungen gut balanciert sind.

\subsection{Prädiktive Effekte des Migrationshintergrundes}

Die Schätzung der prädiktiven Effekte des Migrationshintergrundes auf Einstellungen bezüglich heterogener Lerngruppen erfolgte anhand von Random-Intercept-Modellen. Dabei wurde für jede Dimension der abhängigen Variable zunächst ein Modell spezifiziert, das auf Ebene 1 (within-person Ebene) effektkodierte Indikatorvariablen für die Art der Heterogenität 
enthielt. Dieses Modell wurde dann um einen dummykodierten Ebene-2-Prädiktor des Migrationshintergrundes erweitert. Dessen Regressionsgewicht kann aufgrund der Effektkodierung auf Ebene 1 als prädiktiver Effekt des Migrationshintergrundes auf die personenspezifische mittlere Ausprägung der jeweiligen Dimension der Einstellungen (über die Arten der Heterogenität hinweg) interpretiert werden.

Tabelle 3. Standardisierte Effekte der Random-Intercept-Modelle.

\begin{tabular}{|c|c|c|c|c|c|c|c|c|c|c|}
\hline & \multicolumn{2}{|c|}{ Wert } & \multicolumn{2}{|c|}{ Kosten } & \multicolumn{2}{|c|}{ negative Emotionen } & \multicolumn{2}{|c|}{$\begin{array}{l}\text { wahrgenommene } \\
\text { Kompetenz }\end{array}$} & \multicolumn{2}{|c|}{$\begin{array}{l}\text { intrinsische } \\
\text { Motivation }\end{array}$} \\
\hline & M1 & M2 & M3 & M4 & M5 & M6 & M7 & M8 & M9 & M10 \\
\hline Intercept & .00 & -.03 & .00 & $.09 *$ & .00 & $.10 *$ & .00 & $-.15 * *$ & .00 & -.09 \\
\hline $\mathrm{I}^{\mathrm{Soz}}$ & $.32 * * *$ & $.32 * * *$ & $-.13 * * *$ & $-.13 * * *$ & $-.13 * *$ & $-.13 * * *$ & -.01 & -.01 & $.28 * * *$ & $.28 * * *$ \\
\hline $\mathrm{I}^{\mathrm{Lei}}$ & $-.30 * * *$ & $-.30 * * *$ & $.41 * * *$ & $.41 * * *$ & $.35 * * *$ & $.35 * * *$ & $-.08 *$ & $-.08 *$ & $-.17 * * *$ & $-.17 * * *$ \\
\hline $\mathrm{I}^{\mathrm{MGH}}$ & & .10 & & $-.30 * *$ & & $-.32 * * *$ & & $.41 * * *$ & & $.26^{*}$ \\
\hline$\sigma^{2}\left(e_{i j}\right)$ & .542 & .542 & .625 & .625 & .510 & .510 & .415 & .415 & .452 & .452 \\
\hline$\sigma^{2}\left(u_{j}\right)$ & .394 & .394 & .291 & .625 & .431 & .406 & .582 & .543 & .510 & .495 \\
\hline$R_{c}^{2}$ & .065 & .067 & .086 & .109 & .061 & .087 & .005 & .046 & .040 & .057 \\
\hline$R_{m}^{2}$ & .459 & .460 & .376 & .383 & .491 & .492 & .585 & .586 & .549 & .550 \\
\hline Devianz & 2645.36 & 2643.97 & 2693.13 & 2677.03 & 2617.91 & 2602.79 & 2540.15 & 2520.19 & $\begin{array}{c}2569.0 \\
2\end{array}$ & 2560.40 \\
\hline$p$-Wert & & .394 & & .0001 & & .0001 & & $<.0001$ & & .003 \\
\hline
\end{tabular}

Anmerkungen: Die Fixed und Random Effects stellen die nach den Regeln von Rubin (1987) gepoolten

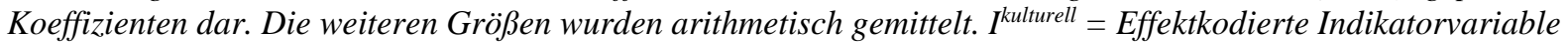
für kulturelle Heterogenität; I leistungsbezogen = Effektkodierte Indikatorvariable für leistungsbezogene Heterogenität; $I^{M H G}=$ Dummykodierte Indikatorvariable: Migrationshintergrund vorhanden (Referenzkategorie: kein Migrationshintergrund); $R_{c}^{2} / R_{m}^{2}$ Determinationkoeffizienten nach Nakagawa und Schielzeth (2013); -Wert stellt das Ergebnis eines $\chi^{2}$-Differenzentest mit dem jeweiligen genesteten Modell ohne den Prädiktor IMHG dar. *p < $.05, * * p<.01, * * * p<.001$.

Die Ergebnisse (siehe Tabelle 4) zeigen zunächst, dass die befragten Lehramtsstudierenden durchaus zwischen den verschiedenen Arten von Heterogenität differenzieren. Mit Ausnahme der Dimension „wahrgenommene Kompetenz“ sind alle Ebene-1-Prädiktoren signifikant und von bedeutsamer Größe, wobei sich ein einheitliches Muster zeigt: Bei positiv gepolten Dimensionen (Wert und intrinsische Motivation) erhält die kulturelle Heterogenität wesentlich höhere Zustimmung als die soziale und leistungsbezogene, während es sich bei den negativ konnotierten Dimensionen (Kosten und negative Emotionen) umgekehrt verhält.

Die Regressionsgewichte des Ebene-2-Prädiktors I ${ }^{\mathrm{MGH}}$ zeigen an, dass die Studierenden mit Migrationshintergrund die signifikant positiveren Überzeugungen $\mathrm{zu}$ heterogenen Lerngruppen aufweisen: Sie zeigen signifikant geringere Kostenüberzeugungen und negative Emotionen, schreiben sich jedoch eine höhere Kompetenz und Motivation für den Umgang mit heterogenen Lerngruppen zu. Die Effektstärken bewegen sich dabei im Bereich kleiner bis moderater Größe nach der Taxonomie von Cohen (1988). Lediglich für die Dimension „Wert“ 
finden sich keine signifikanten Unterschiede zwischen Lehramtsstudierenden mit und ohne Migrationshintergrund.

\section{Diskussion}

Ziel dieser Studie war die Beantwortung der Frage, inwiefern Lehramtsstudierende mit Migrationshintergrund differentielle Überzeugungen zu heterogenen Lerngruppen aufweisen. Dazu wurden anhand eines Matchingverfahrens zwei in vielen relevanten Kovariaten vergleichbare Gruppen von Studierenden mit und ohne Migrationshintergrund gebildet. Die Ergebnisse der darauffolgenden Analysen zeigen: Lehramtsstudierenden mit Migrationshintergrund haben tatsächlich günstigere Überzeugungen (geringere Kosten, höhere Motivation, weniger negative Emotionen und höhere wahrgenommene Kompetenz) als solche ohne Migrationshintergrund. Lediglich in der Dimension der Wertschätzung heterogener Lerngruppen zeigen sich keine signifikanten Unterschiede. Damit können ein Stück weit auch die Ergebnisse beispielsweise von Hachfeld und Kollegen (2012) bestätigt werden, die multikulturelle Überzeugungen häufiger bei Lehramtsanwärterinnen und -anwärtern mit Migrationshintergrund fanden.

Die höhere berichtete intrinsische Motivation in Bezug auf den Umgang mit Heterogenität bei Lehramtsstudierenden mit Migrationshintergrund könnte erklären, weshalb diese auch ein stärkeres Engagement im Umgang mit bzw. eine stärkere Förderung von Schülerinnen und Schülern, die ebenfalls Migrationsgeschichte haben, zeigen bzw. betreiben (Georgi, Karakas \& Ackermann, 2011).

Die vorliegende Evidenz für differentielle Überzeugungsprofile von Lehramtsstudierenden mit Migrationshintergrund gegenüber heterogenen Lerngruppen kann natürlich nicht direkt zur Bewertung der bildungsadministrativen Maßnahme bzw. für die daraus resultierende normative politisch-administrative Entscheidung herangezogen werden, gezielt Menschen mit Migrationshintergrund für den Lehrerinnen- und Lehrerberuf zu gewinnen, da sie lediglich einen spezifischen Aspekt einer langen hypothetischen Wirkungskette ausgehend von Einstellungen von Lehrpersonen über deren Handlungen bis hin zu deren Wirkungen untersucht. Vielmehr konnte ein erster Baustein (differente Einstellungen) zur Beantwortung dieser größeren Frage beigetragen werden.

Die Erkenntnis um die sich aufgrund der Ergebnisse nahelegende Relevanz des Merkmals „Migrationshintergrund“ kann zum Anlass genommen werden, nicht nur wie bisher über die Frage der Rekrutierung von Lehramtsstudierenden mit Migrationshintergrund nachzudenken, sondern (auch) über die Einstellungen $\mathrm{zu}$ heterogenen Lerngruppen aller 
(angehenden) Lehrpersonen und deren Reflexion in der Lehrerbildung (einschließlich Fortund Weiterbildung) (Smith, 2015): Werden Fragen der Selbstwirksamkeit auch auf Einstellungen übertragen, wäre z.B. zu erwarten, dass gelungene und positive Praxiserfahrungen mit Blick auf heterogenen Lerngruppen die mitgebrachten Einstellungen positiv beeinflussen. Hierzu bedarf es jedoch einer für diese Frage sensiblen Betreuungs- und Reflexionspraxis im Rahmen schulpraktischer Professionalisierung.

Die verstärkte Anwerbung und Qualifikation von angehenden Lehrpersonen mit Migrationshintergrund im Sinne einer zielgruppenspezifischen Rekrutierungsmaßnahme (selektierenden Akquise) erscheint vor dem Hintergrund der hier berichteten Ergebnisse sinnvoll, müsste folglich jedoch durch einschlägige Lehrerbildungsmaßnahmen im Sinne einer umfassenderen interkulturellen Öffnung von Schule (Karaksoglu, 2011) ergänzt werden, wie dies etwa im anglophonen Raum durch zahlreiche Initiativen im Bereich der multi-culturalteacher-education (Cochran-Smith, Davis \& Fries, 2004; Rao, 2005) unternommen wird.

Für künftige Forschung wäre es spannend, ähnliche Fragestellungen an im Dienst stehenden Lehrkräften durchzuführen, um $\mathrm{zu}$ untersuchen, inwiefern der Migrationshintergrund auch in dieser biographischen Phase mit den Einstellungen assoziiert ist. Ebenso könnte dann auch der Frage nachgegangen werden, inwiefern beispielsweise Migrationshintergrund, Einstellungen und das tatsächliche Lehrerhandeln zusammenhängen. Zudem spielten in der berichteten Studie Einstellungen zur Mehrsprachigkeit von Schülerinnen und Schülern keine Rolle, obgleich auch diese Form von Heterogenität eine besondere Herausforderung für Lehrkräfte darstellt (Fischer, 2018).

\section{Literatur}

Adelson, J. L. (2013). Educational Research with Real-World Data: Reducing Selection Bias with Propensity Scores. Practical Assessment, Research \& Evaluation, 18(15), 1-11.

Ajzen, I. \& Fishbein, M. (2000). Attitudes and the Attitude-Behavior Relation: Reasoned and Automatic Processes. European Review of Social Psychology, 11(1), 1-33.

Akbaba, Y., Bräu, K. \& Zimmer, M. (2013). Erwartungen und Zuschreibungen - eine Diskursanalyse und kritische Reflexion der bildungspolitischen Debatte. In K. Bräu, V. Georgi, Y. Karakasoblu \& K. Rotter (Hrsg.), Lehrerinnen und Lehrer mit Migrationshintergrund (S. 37-58). Münster u.a.: Waxmann.

Avramidis, E. \& Norwich, B. (2002). Teacher's attitudes towards integration/inclusion: A 
review of the literature. European Journal of Special Needs Education, 17, 129-147.

Bandorski, S. \& Karakasoglu, Y. (2013). Macht ,Migrationshintergrund‘ einen Unterschied? Studienmotivation, Ressourcen und Unterstützungbedarf von Lehramtsstudierenden mit und ohne Migrationshintergrund. In K. Bräu, V.B. Georgi, Y. Karakasoglu \& C. Rotter (Hrsg.), Lehrerinnen und Lehrer mit Migrationshintergrund. Zur Relevanz eines Merkmals in Theorie, Empirie und Praxis (S. 133-156). Münster u.a.: Waxmann.

Bates, D., Maechler, M. \& Bolker, B. (2013). Ime4: Linear Mixed-Effects Models Using S4 Classes. Verfügbar unter: http://cran.r-project.org/package=lme4 [02.10.2018].Baumert, J. \& Lehmann, R. (1997). TIMSS - Mathematisch-naturwissenschaftlicher Unterricht im internationalen Vergleich. Opladen: Leske+Budrich.

Berthold, C. \& Leichsenring, H. (Hrsg.). CHE Diversity Report. C2 Lehramt. Verfügbar unter: https://www.che-consult.de/fileadmin/pdf/publikationen/CHE_Diversity_Report_C2.pdf [29.01.2019].

Bildungsberichterstattung, Autorengruppe (2016). Bildung in Deutschland 2016. Verfügbar unter: http://www.uni-heidelberg.de/md/journal/2016/12/bildungsbericht_2016.pdf [02.08.2018].

Blossfeld, H.-P. et al. (Hrsg.) (2007). Bildungsgerechtigkeit. Jahresgutachten 2007. Wiesbaden: VS Verlag.

Bohl. T., Budde, J. \& Rieger-Ladich, M. (Hrsg.) (2017). Umgang mit Heterogenität in Schule und Unterricht. Bad Heilbrunn: Klinkhardt/UTB.

Bos, W., Hornberg, S., Arnold, K.-H., Faust, G., Fried, L., Lankes, E.-M.-, Schwippert, K. \& Valtlin, R. (Hrsg.) (2007). IGLU 2006. Lesekompetenz von Grundschulkindern und Deutschland und im internationalen Vergleich. Münster: Waxmann.

Bosse, S. \& Spörer, N. (2014). Erfassung der Einstellung und der Selbstwirksamkeit von Lehramtsstudierenden zum inklusiven Unterricht. Empirische Sonderpädagogik, 6(4), 279299.

Bräu, K., Georgi, V.B., Karakasoglu, Y. \& Rotter, C. (2013). Einleitung. In K. Bräu, V.B. Georgi, Y. Karakasoglu \& C. Rotter (Hrsg.), Lehrerinnen und Lehrer mit Migrationshintergrund. Zur Relevanz eines Merkmals in Theorie, Empirie und Praxis (S. 7-13). Münster u.a.: Waxmann.

Bourdieu, P. (2012). Ökonomisches Kapital kulturelles Kapital, soziales Kapital. In U. Bauer, U. Bittlingmayer \& A. Scherr (Hrsg.), Handbuch Bildungs- und Erziehungssoziologie (S. 229-242). Berlin: Springer.

Budde, J. (2012). Problematisierende Perspektiven auf Heterogenität als ambivalentes Thema 
der Schul- und Unterrichtsforschung. Zeitschrift für Pädagogik, 58(4), 522-540.

Cochran-Smith, M., Davis, D., \& Fries, K. (2004). Multicultural teacher education: research, practice, and policy. In J. Banks \& C. Banks (Eds.), Handbook of research on multicultural education (2, pp. 931-975). San Francisco: Jossey-Bass.

Cohen, J. (1988). Statistical Power Analysis for the Behavioral Sciences (2 ${ }^{\text {nd }}$. Ed.). Hillsdale, N.J.: L. Erlbaum Associates.

Cramer, C. (2016). Forschung zum Lehrerinnen- und Lehrerberuf. Systematisierung und disziplinäre Verortung eines weiten Forschungsfeldes. Bad Heilbrunn: Klinkhardt.

Cunningham, M. \& Hargreaves, L. (2007). Minority Ethnic Teachers' Professional Experiences. Evidence from the Teacher Status Project. Research Report RR853. Verfügbar unter: http://www.ssg-bildung.ub.unierlangen.de/Minority_Ethnic_Teachers_Professional.pdf [02.10.2018].

Dorman, J. P. (2008). The Effect of Clustering on Statistical Tests: An Illustration Using Classroom Environment Data. Educational Psychology Review, 28(5), 583-595.

Eagly, A. H. \& Chaiken, S. (1993). The psychology of attitudes. Fort Worth, TX: Harcourt Brace Jovanovich College Publishers.

Edelmann, D. (2008). Pädagogische Professionalität im transnationalen sozialen Raum. Eine qualitative Untersuchung über den Umgang von Lehrpersonen mit der migrationsbedingten Heterogenität ihrer Klassen. Berlin: LIT-Verlag.

Edelmann, D. (2013). Lehrkräfte mit Migrationshintergrund - ein Potenzial pädagogischer Professionalität im Umgang mit migrationsbedingter Heterogenität. In K. Bräu, V.B. Georgi, Y. Karakasoglu \& C. Rotter (Hrsg.), Lehrerinnen und Lehrer mit Migrationshintergrund. Zur Relevanz eines Merkmals in Theorie, Empirie und Praxis (S. 197-208). Münster u.a.: Waxmann.

Fischer, N. (2018). Professionelle Überzeugungen von Lehrkräften - vom allgemeinen Konstrukt zum speziellen Fall von sprachlich-kultureller Heterogenität in Schule und Unterricht. Psychologie in Erziehung und Unterricht, 65(1), 35-51.

Fives, H. \& Buehl, M. M. (2012). Spring cleaning for the construct of teachers' beliefs. In A. Kaplan (Ed.), Educational Psychology Handbook (pp. 471-499). APA.

Ganzeboom, H.B.G., De Graaf, P.M., Treiman, D.J. \& De Leeuw, J. (1992). A standard international socio-economic index of occupational status. Social Science Research, 21(1), $1-56$.

Gebauer, M. M., McElvany, N. \& Klukas, S. (2013). Einstellungen von Lehramtsanwärterinnen und Lehramtsanwärtern zum Umgang mit heterogenen 
Schülergruppen in Schule und Unterricht. Jahrbuch der Schulentwicklung, 17, 191-216.

Georgi, V. B. (2011). Theoretischer Bezugsrahmen der Studie und Stand der Forschung zu Lehrenden mit Migrationshintergrund und minority teachers. In V.B. Georgi, L. Ackermann \& N. Karakaş (Hrsg.), Vielfalt im Lehrerzimmer. Selbstverständnis und schulische Integration von Lehrenden mit Migrationshintergrund in Deutschland (S. 1643). Münster u.a.: Waxmann.

Georgi, V.B., Ackermann, L. \& Karalas, N. (2011). Vielfalt im Lehrerzimmer. Selbstverständnis und schulische Intergration von Lehrenden mit Migrationshintergrund in Deutschland. Münster u.a.: Waxmann.

Georgi, V.B. (2013). Empirische Forschung zu Lehrenden mit Migrationshintergrund, minority teachers und teachers of color. In K. Bräu, V.B. Georgi, Y. Karakasoglu \& C. Rotter (Hrsg.), Lehrerinnen und Lehrer mit Migrationshintergrund. Zur Relevanz eines Merkmals in Theorie, Empirie und Praxis (S. 85-103). Münster u.a.: Waxmann.

Graham, J. W., Cumsille, P. E. \& Elek-Fisk, E. (2003). Methods for Handling Missing Data. In Schinka, J. A., Velicer, W. F. (Eds.), Handbook of Psychology (2 ${ }^{\text {nd }}$. Ed., pp. 87-114). Hoboken, NJ: John Wiley \& Sons, Inc.

Hachfeld, A., Schroeder, S., Anders, Y., Hahn, A., \& Kunter, M. (2012). Multikulturelle Überzeugungen. Herkunft oder Überzeugungen? Welche Rolle spielen der Migrationshintergrund und multikulturelle Überzeugungen für das Unterrichten von Kindern mit Migrationshintergrund. Zeitschrift für Pädagogische Psychologie, 26(2), 101120. doi:10.1024/1010-0652/a000064

Hartinger, A., Kleickmann, T. \& Hawelka, B. (2006). Der Einfluss von Lehrervorstellungen zum Lernen und Lehren auf die Gestaltung des Unterrichts und auf motivationale Schülervariablen. Zeitschrift für Erziehungswissenschaft, 9(1), 110-126.

Hartwig, S. J., Schwabe, F., Gebauer, M.M. \& McElvany, N. (2017). Wie beurteilen Lehrkräfte und Lehramtsstudierende Leistungsheterogenität? Ausprägungen, Zusammenhänge und Prädiktoren von Einstellungen und Motivation. Psychologie in Erziehung und Unterricht, 64(2), 94-108.

Henry, A. (2018). Exploring Minority Teachers' Experiences Pertaining to Their Value in Education: A Single Case Study of Teachers in New York City. Dissertation. Northcentral University.

Heyl, V., Janz, F., Seifried, S. \& Trumpa, S. (2014). Einstellungen zu Inklusion von Lehrkräften und Eltern an der Grundschule. In E. Franz, S. Trumpa \& I. Esslinger-Hinz (Hrsg.), Inklusion. Eine Herausforderung für die Grundschulpädagogik. Ein Studienbuch zu 
grundlegenden Themenfeldern der Pädagogik (S. 200-211). Schneider Verlag: Hohengehren.

Ho, D. E., Imai, K., King, G. \& Stuart, E. A. (2007). Matching as Nonparametric Preprocessing for Reducing Model Dependence in Parametric Causal Inference. Political Analysis: An Annual Publication of the Methodology Section of the American Political Science Association, 15(3), 199-236. Oxford University Press.

Hox, J. J. (2010). Multilevel Analysis: Techniques and Applications (2 ${ }^{\text {nd }}$ Ed.). New York: Routledge.

Karakaş, N. (2011). Benachteiligungs- und Diskriminierungserfahrungen. In V.B. Georgi, L. Ackermann \& N. Karakaş (Hrsg.), Vielfalt im Lehrerzimmer. Selbstverständnis und schulische Integration von Lehrenden mit Migrationshintergrund in Deutschland (S. 214241). Münster u.a.: Waxmann.

Karakasoglu, Y. (2011). Lehrer, Lehrerinnen und Lehramtsstudierende mit Migrationshintergrund. Hoffnungsträger der interkulturellen Öffnung von Schule. In Neumann, U. \& Schneider, J. (Hrsg.), Schule mit Migrationshintergrund (S. 121-135). Münster u.a.: Waxmann.

Kohli, R. (2018). Behind School Doors: The Impact of Hostile Racial Climates on Urban Teachers of Color. Urban Education, 53(3), 307-333.

Kopmann, H. \& Zeinz, H. (2016). Lehramtsstudierende und Inklusion - Einstellungsbezogene Ressourcen, Belastungsempfinden in Hinblick auf unterschiedliche Förderbedürfnisse und Ideen zur Individualförderung. Zeitschrift für Pädagogik, 62(2), S. 263-281.

Kunter, M. \& Pohlmann, B. (2009). Lehrer. In Wild, E. (Hrsg.), Pädagogische Psychologie (S. 261-282). Berlin: Springer.

Kunter, M., Baumert, J., Blum, W., Klusmann, U., Krauss, S. \& Neubrand, M. (2011). Professionelle Kompetenz von Lehrkräften. Ergebnisse des Forschungsprogramms COACTIV. Münster u.a.: Waxmann.

Kunz, A., Luder, R. \& Moretti, M. (2010). Die Messung von Einstellungen zur Inklusion. Empirische Sonderpädagogik, 2(3), 83-94.

Magaldi, D., Conway, T. \& Trub, L. (2018). "I Am Here for a Reason": Minority Teachers Bridging Many Divides in Urban Education. Race, Ethnicity and Education, 21(3), 306-318. Mehta, P. D. \& Neale, M. C. (2005). People Are Variables Too: Multilevel Structural Equations Modeling. Psychological Methods, 10(3), 259-284.

Merk, S., Cramer, C., Dai, N., Bohl, T. \& Syring M. (2018). Faktorielle Validität der Einstellungen von Lehrkräften $\mathrm{zu}$ heterogenen Lerngruppen. Journal of Educational 
Research Online (JERO), 10(2), 34-53.

Nakagawa, S. \& Schielzeth, H. (2013). A General and Simple Method for Obtaining R2 from Generalized Linear Mixed-Effects Models. Methods in Ecology and Evolution / British Ecological Society, 4(2), 133-142.

Neugebauer, M. \& Klein, O. (2016). Profitieren Kinder mit Migrationshintergrund von pädagogischen Fachkräften mit Migrationshintergrund? Kölner Zeitschrift für Soziologie und Sozialpsychologie, 68(2), (S. 259-283).

Otyakmaz, B.Ö. (2004). Dequalifizierung von Professionellen mit Migrationshintergrund im psychosozialen Arbeitskontext. In Y. Karakasoglu \& J. Lüddecke (Hrsg.), Migrationsforschung und interkulturelle Pädagogik (S. 117-130). Münster u.a.: Waxmann.

Pajares, M. F. (1992). Teachers' Beliefs and Educational Research: Cleaning up a messy construct. Review of Educational Research, 62(3), (pp. 307-332).

Prenzel, M., Artelt, C., Baumert, J., Blum, W., Hammann, M., Klieme, E \& Pekrun, R. (2006). PISA 2006. Ergebnisse der dritten internationalen Vergleichsstudie. Münster: Waxmann. Presse- und Informationsamt der Bundesregierung (2007). Nationaler Integrationsplan. Neue Wege - Neue Chancen. Verfügbar unter: https://www.bundesregierung.de/Content/DE/Archiv16/Artikel/2007/07/Anlage/200708-30-nationaler-integrationsplan.pdf?_blob=publicationFile\&v=1 [02.10.2018].

R Core Team. (2014). R: A Language and Environment for Statistical Computing. Vienna, Austria. Verfügbar unter: http://www.r-project.org/ [02.08.2018].

Rao, S. (2005). Effective Multicultural Teacher Education Programs: Methodological and Conceptual Issues. Education, 126(2), 279-291.

Rosenbaum, P. R. \& Rubin, D. B. (1983). The Central Role of the Propensity Score in Observational Studies for Causal Effects. Biometrika, 70(1), 41-55.

Rosenberg, M. J. \& Hovland, C. I. (1960). Cognitive, affective and behavioral components of attitudes. In Hovland, C. I. \& Rosenberg, M. J. (Eds.), Attitude organization and change. An analysis of consistency among attitude components (tape 3, pp. 1-14). New Haven: Yale University Press.

Rotter, C. (2012). Lehrkräfte mit Migrationshintergrund. Individuelle Umgangsweisen mit bildungspolitischen Erwartungen. Zeitschrift für Pädagogik, 58(2), 204-222.

Rubin, D. B. (1987). Multiple Imputation for Nonresponse in Surveys. Wiley Series in Probability and Mathematical Statistics. Applied Probability and Statistics. New York: Wiley.

Sachverständigenrat deutscher Stiftungen für Integration und Migration (Hrsg.) (2016). 
Doppelt benachteiligt? Kinder und Jugendliche mit Migrationshintergrund im deutschen Bildungssystem. Eine Expertise im Auftrag der Stiftung Mercator. Verfügbar unter: https://www.stiftung-

mercator.de/media/downloads/3_Publikationen/Expertise_Doppelt_benachteiligt.pdf [04.02.2019].

Schäfer, T. \& Brückner, G. (2008). Soziale Homogenität der Bevölkerung bei alternativen Definitionen für Migration. Eine Analyse am Beispiel der Bildungsbeteiligung, Erwerbstätigkeit und Einkommen auf der Basis von Mikrozensusdaten. Wirtschaft und Statistik, 12, 1046-1066.

Smith, T.-J. (2015). Lehrkräfte mit Zuwanderungsgeschichte als Change Agents. Wie Diversity im Lehrerzimmer realisiert werden kann. In A. Holzbrecher \& U. Over (Hrsg.), Handbuch Interkulturelle Schulentwicklung (S. 207-213). Weinheim: Beltz.

Statistisches Bundesamt (2008). Anteil der Personen mit Migrationshintergrund unter den Erwerbstätigen mit Hochschulabschluss und unter den Lehrkräften in 2008 in Deutschland. Verfügbar unter: https://de.statista.com/statistik/daten/studie/159409/umfrage/personenmit-migrationshintergrund-bei-lehrkraeften/ [29.01.2019].

Statistisches Bundesamt (2017). Bevölkerung und Erwerbstätigkeit, Bevölkerung mit Migrationshintergrund - Ergebnisse des Mikrozensus. Fachserie 1, Reihe 2.2. Verfügbar unter:

https://www.destatis.de/DE/Publikationen/Thematisch/Bevoelkerung/MigrationIntegration /Migrationshintergrund.html [02.10.2018].

Strasser, J. \& Steber, C. (2009). Lehrerinnen und Lehrer mit Migrationshintergrund - Eine empirische Reflexion einer bildungspolitischen Forderung. In J. Hagedorn, V. Schurt, C. Steber \& W. Waburg (Hrsg.), Ethnizität, Geschlecht, Familie und Schule (S. 97-126). Wiesbaden: VS Verlag.

Syring, M., Tillmann, T., Weiß, S. \& Kiel, E. (2018). Positive Einstellung zur Inklusion ablehnende Haltung zur Umsetzung in der Schule: Analyse des Widerspruchs durch Überprüfung eines aus der Heterogenitätsforschung adaptierten Messinstruments für die Inklusion an Lehramtsstudierenden. Psychologie in Erziehung und Unterricht, 65(3), 206220.

Thoemmes, F. J. \& Kim, E. S. (2011). A Systematic Review of Propensity Score Methods in the Social Sciences. Multivariate Behavioral Research, 46(1), 90-118.

Verband Bildung und Erziehung (2006). Interkulturelle Herausforderungen pädagogisch begegnen. Positionspapier, 11. November 2006. Verfügbar unter: http://vbe.de/880.html 
[02.10.2018].

Voss, T., Kleickmann, T., Kunter, M. \& Hachfeld, A. (2011). Überzeugungen von Mathematiklehrkräften. In Baumert, J., Blum, W., Kunter M. \& Neubrand, M. (Hrsg.), Professionelle Kompetenz von Lehrkräften. Ergebnisse des Forschungsprogramms COACTIV (S. 235--257). Münster u.a.: Waxmann.

Voss, T., Kunina-Habenicht, O., Hoehne, V. \& Kunter, M. (2015). Stichwort Pädagogisches Wissen von Lehrkräften: Empirische Zugänge und Befunde. Zeitschrift für Erziehungswissenschaft, 18(2), 187-223. 